\title{
Value Analysis and Realization of Artistic Intervention in Rural Revitalization Based on the Fuzzy Clustering Algorithm
}

\author{
Jianhui Liang (iD \\ Guangzhou Academy of Fine Arts, Guangzhou 510006, China \\ Correspondence should be addressed to Jianhui Liang; liangjh@gzarts.edu.cn
}

Received 16 December 2021; Revised 2 January 2022; Accepted 3 January 2022; Published 25 January 2022

Academic Editor: Tongguang Ni

Copyright (c) 2022 Jianhui Liang. This is an open access article distributed under the Creative Commons Attribution License, which permits unrestricted use, distribution, and reproduction in any medium, provided the original work is properly cited.

\begin{abstract}
Intervening in the revitalization and development of art villages is one of the ways to build regional brands in rural areas, fit in with contemporary aesthetics, and meet the needs of modern people for diversified life. At the same time, it is not limited by artistry itself, but a comprehensive artistic activity with modernity, industrial linkage, and sustainability. This paper uses the HPR (Hestenes-Powell-Rockafellar) multiplier method to solve and establishes a SSFCM-HPR (semisupervised fuzzy C-means clustering based on HPR) algorithm. The experimental results on datasets show that FCM-UserCF (fuzzy C-means-user collaborative filtering) algorithm effectively solves the problem of data sparsity and improves the accuracy of recommendation. Rural planning and design under the intervention of art is a kind of respect for local memory, an inheritance of folk crafts, a reshaping of history and culture, and an identification with the countryside itself. It also puts forward new development ideas and guiding methods for the sustainable development of rural areas.
\end{abstract}

\section{Introduction}

Rural areas, which are China's most difficult and pressing issue, are attempting to reshape rural order, protect ancient buildings in rural areas, and revive rural culture through art intervention. Rural revitalization has been a top priority of the Chinese government policy in recent years, and it is expected to continue to do so in the future. At the government level, China has issued a series of related policies in recent years, demonstrating the country's commitment and attitude toward rural revitalization. A new wave of development and construction has erupted in domestic villages, guided by the national rural revitalization strategy. Not only for rural material construction but also for multidisciplinary participation [1], creating beautiful countryside is a difficult and long-term task.

With the development of modernization and towns, rural society is facing the strong invasion of external modern culture, and there is also an endogenous cultural crisis. In the cultural environment of "coexistence of old and new," there are some problems in rural community culture, such as insufficient endogenous development power, insufficient innovation ability, declining vitality, and unsustainable development of traditional culture $[2,3]$. Since the rural revitalization strategy was put forward, all parts of the country have responded one after another and continued to promote all-round inheritance and promotion of rural infrastructure construction, ecological environment management, and rural cultural custom protection [4]. Intervening in the revitalization and development of art villages is one of the ways to build regional brands in rural areas, fit in with contemporary aesthetics, and meet the needs of modern people for diversified life [5]. The uniqueness of contemporary Chinese villages is being dispelled, the traditional culture is being impacted, and the living environment has not been paid attention to and improved; instead, it has become an activity for artists to entertain themselves.

Involved in rural revitalization of art, it has unique advantages in creation design, ingenuity culture, cultural protection, and dissemination $[6,7]$. Giving full play to the role of art is the inherent requirement of rural revitalization, and the involvement of art in rural construction has become the main practice of rural construction in China. Based on this, this paper explores the strategy of artistic mediation in 
rural design under the background of rural revitalization. This paper puts forward the value analysis and realization path of artistic intervention in rural revitalization based on FC (fuzzy clustering) algorithm, aiming at awakening villagers' cultural consciousness in cultural construction.

Based on the analysis and deduction of the four elements of the festival, this paper points out that, to meet the inherent demands of the four elements in the festival, the balance of each element can only be achieved through the corresponding linkage mechanism, which is also the necessary way to successfully hold the festival. The linkage mechanism is dynamic and flexible, which acts on the festival and also makes the festival produce different functions, meanings, and achievements under different backgrounds and conditions.

\section{Related Work}

How to involve the art festival in rural revitalization is not only a domestic topic but also a common concern of the whole world. If the scope discussed in this topic is further expanded-which is actually necessary-it should be extended to cities. In [8], from the perspective of social ecology, it is considered that the current rural areas present a "weak niche." Xu [9] used scientific experiments and stories to prove the practical "work" way of science and held that the so-called "culture" actually exists in a dynamic and constant "action." "Before the conflict with other people, no one lives in a certain ' culture,' enjoys a certain ' paradigm,' or belongs to a certain society. The emergence of these words is the result of expanding the network and crossing the alien crowd." Pan [10] made comprehensive statistics and research on the economic role, indirect benefits, comprehensive tourism income, and jobs provided by the art festival. Zhang et al. [11] presented a detailed description and evaluation of the public art and Art Festival on the coast. Shang [12] analyzed the development and ways of the creative industry through the case of ancient town drama festival.

The goal of clustering analysis is to divide a wide range of items into distinct categories (clusters). The things in each cluster have some similarities, and the things in different clusters have some differences. Clustering has been proven to be effective in a variety of scenarios and is an important research direction in big data mining. A new metric space based on the fuzzy induction topology was established in [13]. Unsupervised distance learning algorithm was proposed in $[14,15]$. Researchers have been working on distance modification for the past two years. An unsupervised distance learning algorithm based on the DI-FCM (doubleindices fuzzy $C$-means) algorithm framework-a doubleindex fuzzy $C$-means algorithm based on hybrid clustering learning HDDI-FCM (double-indices fuzzy $C$-means with hybrid distance) - was proposed in [16]. Karunambigai et al. [17] proposed a fuzzy scatter matrix-based clustering algorithm that aims to minimize the trace of the intraclass fuzzy scatter matrix while maximizing the trace of the interclass fuzzy scatter matrix, integrates hard and soft clustering, and assigns a hard core boundary to each class. A fuzzy kernel clustering algorithm with different attributes weighted in the feature space was proposed in [18], which can effectively deal with the clustering problems of linear inseparability and unbalanced attributes. A fuzzy kernel clustering algorithm based on comprehensive comparative analysis was proposed in [19]. These methods have partially solved the clustering problem for nonspherical distribution data, but the construction and selection of kernel functions, as well as the determination of kernel function parameters, have become new problems for these methods.

\section{Research Method}

3.1. Strategies and Methods of Art Intervention in Rural Planning and Design. As the birthplace of Chinese culture, the countryside bears Chinese excellent folk traditions, unique lifestyle, and behavior habits and is the resort of homesickness and the carrier of historical context. Under the influence of modernization and urbanization, in order to retain homesickness memory and historical context in the process of art intervention in rural planning and design, it is necessary to realize the mutual fit between the new and old environment in the process of art intervention and avoid tough intervention or conservative intervention.

Therefore, in the process of art intervention in rural planning and design, it is necessary to excavate rural local cultural resources to form the accumulation of original materials for art intervention [20]. For the excavation and acquisition of cultural resources, it can collect basic cultural resource materials, pictures and images of rural texture, style, beliefs, folk culture, and traditional skills through indepth interviews, oral statements by the elderly, consulting documents and materials such as county records and rural history, on-the-spot investigation, and experience.

Historical cultural resources, celebrity cultural resources, religious cultural resources, folk cultural resources, and revolutionary cultural resources are the most common types of local cultural resources. To vividly express a variety of local cultural and artistic resources, to provide artistic design elements for art intervention in rural planning and design, and to extract the symbols of cultural elements needed for art to intervene in rural planning and design, it is usually necessary to analyze, classify, and screen the original materials, such as local cultural and artistic resources, combined with the needs of planning and design and the needs of villagers.

Typical local cultural element symbols have been formed after collecting, sorting, classifying, and refining the materials of local cultural resources. Then, using various artistic design techniques, these typical local cultural elements are transformed into "artistic design symbols" with special rural significance and finally applied to artistic intervention in rural planning and design to realize the fit between the new and old environment. The model of strategies and methods involved in artistic rural planning and design is shown in Figure 1.

Involved in artistic rural planning and design, the rural local cultural resources are first excavated through the oral method of the elderly and the method of consulting 


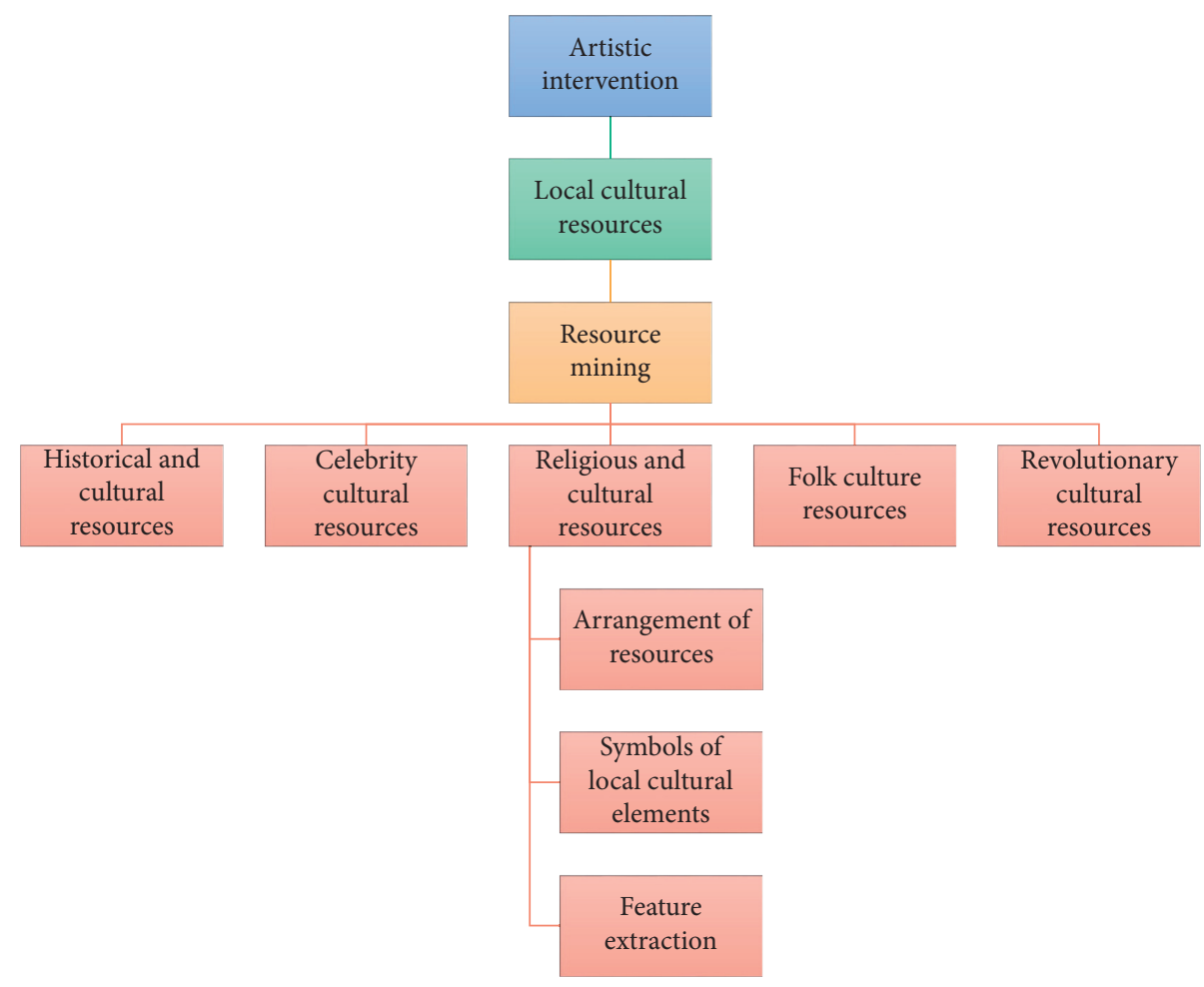

Figure 1: Strategy and method model of artistic rural planning and design.

documents and materials, and then the characteristic and typical local cultural element symbols are extracted by analyzing, classifying, and screening them in conjunction with the design needs and the needs of the villagers in order to complete the planning and design investigation and design analysis. The extracted symbols of local cultural elements are then refined in terms of color, shape, structure, texture, connotation, and other characteristics to form basic artistic design elements, and the local cultural resources are transformed into artistic forms using design techniques such as extension and reproduction, transformation and utilization, integration, and innovation. Finally, the art form is applied to the planning and design of rural art.

3.2. Analysis of the Semisupervised FCM Algorithm for Artistic Intervention. Because the rural culture is formed in the long-term local practice, it has the characteristics of life and locality and has a strong regional character. The cultural consensus formed by the community for a long time is the glue of the rural society, so the cultural break is likely to affect the stability of social order. Therefore, in the process of rural cultural revival, it is necessary to repair the cultural and social fractures caused by man-made in the social transformation, attach importance to the cultural value of local traditions, reshape the rural community through cultural revival, and rebuild the rural community identity.

The rational choice principle of choosing to use more advanced and efficient tools in human social changes cannot be changed, but although the old culture no longer has the tool value, its cultural value has become more important. Therefore, seeing that some valuable local cultures that have recorded human civilization for many years are gradually dying out, it needs to show our attitude and take practical actions to protect, inherit, and develop those most distinctive cultures and skills and record and preserve these living fossils of human civilization for future generations.

FCM (fuzzy $C$-means) is a typical FC algorithm, which applies fuzzy technology to the traditional $C$-means algorithm and has been proved to be one of the most effective clustering methods by practice [21]. However, FCM algorithm needs many iterations in the process of operation, and when the amount of data is particularly large or there are many attributes of data samples, the operation speed will be greatly reduced. The algorithm of SSFCM-HPR (semisupervised fuzzy $C$-means clustering based on HPR (Hestenes-Powell-Rockafellar)) is proposed.

Assume that the number of clustering samples is $n_{s}+n$, among which $n_{s}$ are labeled samples, $n$ are unlabeled samples, and $n_{1}$ belong to the first class, $n_{2}+n_{1}$ belong to the second class, and $n_{s}+n_{s-1}$ belong to the $s$ class. Let the classification number be $c$ and the membership matrix be $U=\left(U^{\prime}, U\right)$.

The expression of distance measurement between the sample and cluster prototype is defined as

$$
\begin{aligned}
\left(d_{i k}\right)^{2} & =\left\|X_{k}-P_{i}\right\| \\
& =\left(X_{k}-P_{i}\right)^{T} W\left(X_{k}-P_{i}\right),
\end{aligned}
$$

where $W$ is the characteristic weighting matrix.

Because all the samples are independent of each other so that the columns of the partition matrix $U^{\prime}$ are independent of each other, one column of the partition matrix $U^{\prime}$ can be chosen as the research object. For the convenience of 
research, the $k$ th supervised sample can be chosen as the research object. Because when $k$ is determined, its corresponding labeled is also determined; that is, the category $j$ is a known quantity.

$$
\begin{aligned}
& \min J_{k}=\sum_{i=1}^{c}\left(u_{i k}^{*}\right)^{m} d_{i k}^{2}, \\
& \text { s.t. }\left\{\begin{array}{l}
\sum_{i=1}^{c} u_{i k}^{*}=1, \\
u_{i k}^{*}+\Delta u-u_{j k}^{*}+y_{i k}^{*}=0, \quad i=1,2, \ldots, c, i \neq j .
\end{array}\right.
\end{aligned}
$$

For unsupervised samples, because the constraint conditions of membership degree have not changed, the formula of membership degree is the same as that of traditional FCM algorithm:

$$
\begin{aligned}
u_{i k}^{*} & =\frac{1}{\sum_{l=1}^{c}\left(d_{i k} / d_{l k}\right)^{2 / m-1}}, \\
k & =1,2, \ldots, n ; \quad i=1,2, \ldots, c .
\end{aligned}
$$

Because the solution of the clustering objective function is an iterative optimization process, if the weight of the $k$ supervised sample is $\omega_{k, k_{1}}$ in the $k_{1}$ iteration, then

$$
\omega_{k, k_{1}}=\frac{u_{j k, k_{1}-1}^{*}}{\max _{i \in\{1, \ldots, c\}, i \neq j}\left(u_{i k, k_{1}-1}^{*}\right)} \text {. }
$$

In the above formula, $j=\underset{i \in\{1, \ldots, c\}}{\operatorname{argmax}}\left(u_{i k, k_{1}-1}^{*}\right)$.

3.3. Intervene in the Realization of Art and Rural Revitalization. Action that is based on participation art intervention, also known as social art intervention, is a type of art intervention that is commonly used in rural areas. Its art is based on field research and refined from the unique regional culture of rural areas, with a stronger emphasis on the intermediary nature of art action and the creation of rural regional culture. Intervening in artistic rural design is to revalue and repair the rural value and reconstruct the relationship between man, nature, and ancestors with the help of rural design, whose core elements are to repair rural production and life, ecosystem, rural social order, and ethical relationship and to promote the emergence and development of new industries and new platforms in rural areas. The restoration of the rural cultural system and ethics, paying attention to the explicit expression and invisible inheritance of rural culture, inspiring public participation and a sense of ownership, and creating a rural space rich in appreciation value are at the heart of artistic intervention in rural design. Art in rural areas not only preserves rural culture's unique value but also facilitates the exchange of rural information and improves the countryside's inclusiveness and openness.

At present, the countryside is facing a serious hollowing phenomenon, and rural public life is gradually declining. In order to revitalize rural public space, artists launched a series of artistic practice activities. By means of art and culture, the symbol resources can be transformed, and a new image of artistic villages and cultural villages with relaxed and rich cultural atmosphere can be created with lightweight rural field art creation. The artistic expression forms include overall planning of rural space, dot space art design, linear street space design, and area space art design. At the same time, reconstruct the villagers' public life, complete the reconstruction of rural capital through collective activities, enhance the display of rural cultural individuality, and promote the rural production and life sustainably.

The basic idea of FCM-UserCF (fuzzy C-means-user collaborative filtering) algorithm designed in this paper is as follows: firstly, FCM algorithm is used to cluster users with similar backgrounds into the same cluster to reduce the dimension of the matrix; then, the data matrix is preprocessed by Slope One algorithm, and the unrated data in the matrix are predicted and filled, so as to solve the disadvantage of the sparse user-item scoring matrix; finally, CF (collaborative filtering) is carried out based on the filled scoring matrix.

The FCM-UserCF algorithm can be disassembled into two steps: step 1: according to the background information of users, use FC to make the background of the same user group similar; step 2: fill in the missing data of the scoring matrix and make recommendations through the UserCF algorithm in CF.

FC algorithm needs to generate a membership matrix, and a fuzzy similarity matrix needs to be built for the data similarity in the matrix [22]. There are many methods to construct the similarity matrix, including the maximumminimum calculation method, cosine angle method, and correlation coefficient method. In this article, the fuzzy similarity matrix $R\left(r_{i j}\right)_{m \times n}$ is constructed by calculating the similarity statistic $r_{i j}$ of data by using the maximum-minimum method, and the formula is

$$
r_{i j}=\frac{\sum_{k=1}^{m} \min \left(x_{i k}{ }^{\prime}, x_{j k}{ }^{\prime}\right)}{\sum_{k=1}^{m} \max \left(x_{i k}{ }^{\prime}, x_{j k}{ }^{\prime}\right)} .
$$

Among them, $i=1,2, \ldots, n ; j=1,2, \ldots, m, n$ represents the number of data pieces, $m$ corresponds to the category that is the number of cluster centers, and $x^{\prime}$ represents the elements in the membership matrix.

Based on all scoring items $I_{u i}=I_{u} \cup I_{i}$ of users $u$ and $i$, if there are missing scoring items in all items, the Slope One algorithm can be used for prediction. Calculate the target user $U$ rating $P\left(u_{j}\right)$ for item $j$.

$$
\begin{aligned}
\operatorname{dev}_{(j, i)} & =\sum_{u \in I \cap J} \frac{u_{j}-u_{i}}{\operatorname{num}(I \cap J)}, \\
p\left(u_{j}\right) & =\frac{I}{\operatorname{num}(R(u) \cap D(j))} \sum_{i \in R(u) \cap D(j)}\left(u_{i}+\operatorname{dev}_{(j, i)}\right) .
\end{aligned}
$$

In the formula $u_{i}, u_{j}$ refers to the true score made by user $U$ on item $i, j$, num $(I \cap J)$ refers to the number of all users who score the intersection of item $i, j, R(u)$ refers to all scoring items of user $U, D(j)$ refers to all average deviation value items calculated with item $j$, and $\operatorname{num}(R(u) \cap D(j))$ refers to the intersection number of all items of $R(u)$ and $D(j)$. 
The FCM-UserCF algorithm proposed in this article needs to cluster the dataset by FCM algorithm according to the user background information so that the user background information within the same class is similar after clustering; Figure 2 shows the specific flow of FCMUserCF.

The planning of traditional rural folk cultural and artistic activities reflects the cultural significance of rural space, explains village characteristics, and influences villagers' daily lives and values. This emphasizes the critical role of traditional rural folk culture and art activities in art intervention in rural planning and design. The goal of planning and developing traditional rural folk culture and art activities is to improve the quality of life for villagers. As a result, the needs of villagers' lives should be the starting point for planning traditional rural folk culture and art activities. In conjunction with the trend of people flow, attention should be paid to the layout and creation of artistic space and cultural creative space, particularly in rural public space and places where people flow gather, as these are the places that can best influence and drive villagers' thought and value identity, as well as promote cultural communication.

Today's rural revitalization needs to pay attention to the long-term investment and long-term interests of rural industries. The goal of rural revitalization is to establish long-term regional brands and regional products that are compatible with them, cross-border, and diversified. The participation of art in regional brand building in rural areas is one of the most direct and effective ways to realize rural revitalization and promote rural sustainable development, and it is also one of the important contents of top-level design in rural revitalization.

\section{Results' Analysis and Discussion}

4.1. Discussion on the Linkage Mechanism of Art Festivals Involved in Rural Revitalization and Development. Under the tide of China's economic development, the cultural ecology is constantly tearing, reorganizing, and constructing, especially in rural areas, where constant cultural acculturation makes the cultural ecology very fragile. However, it is impossible and undesirable to return to the traditional cultural ecology. The festival is a good choice in terms of the feasibility of the measures, the remarkable effect, and the cohesion of the organization.

The last step-by-step process of the festival is not a onestep process. After the completion and implementation of the whole art planning, diverse subjects will have different feedbacks. According to the feedbacks, the content of the festival will be constantly adjusted and enriched, and all of them need to operate the festival sustainably through four linkage mechanisms (Figure 3).

To begin with, the festival's origins are based on a variety of interconnected and contradictory demands from multiple subjects, as well as a need to coordinate problems across subjects through inclusive linkage. Second, the festival's surroundings are important amenities that contribute to the festival's uniqueness. As a result, strengthening the linkages

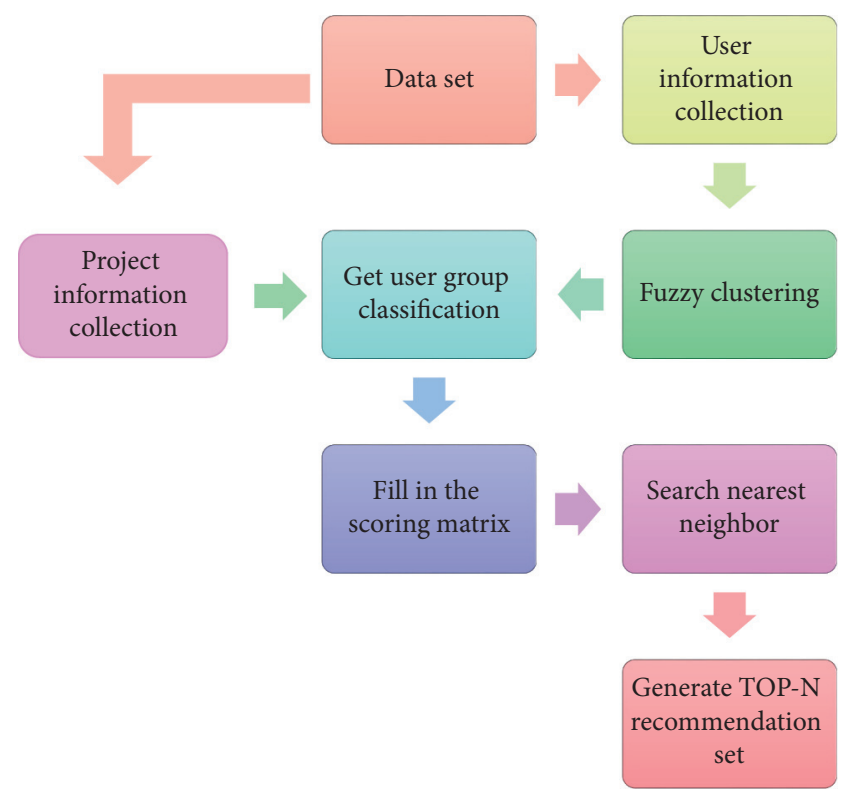

FIgURE 2: Flowchart of FCM-UserCF algorithm.

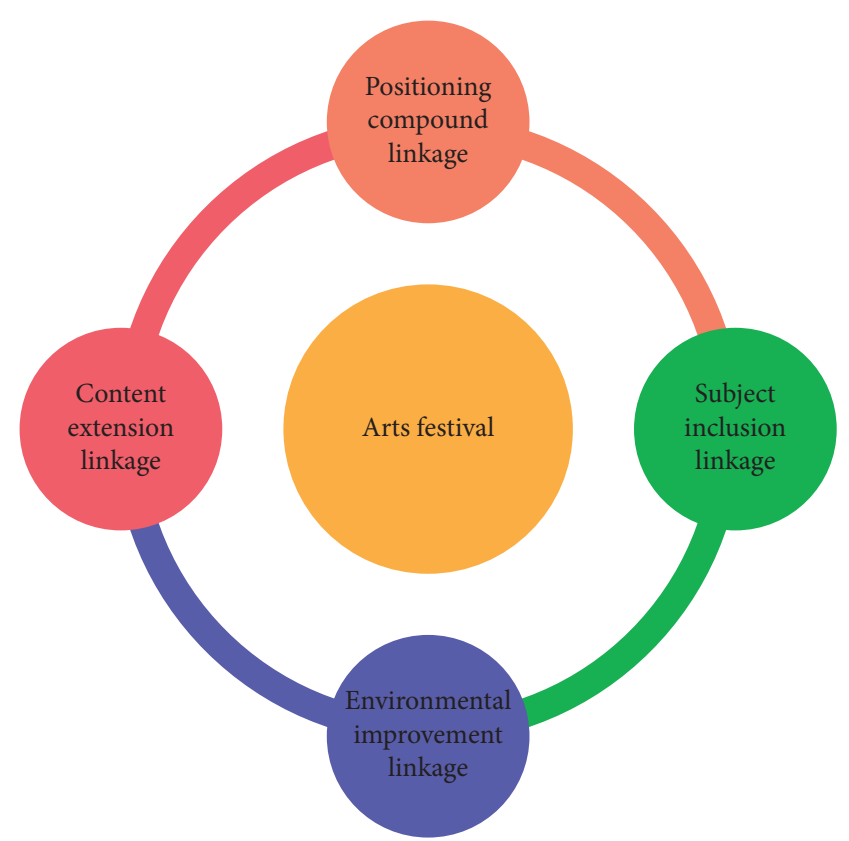

FIgURE 3: Illustration of the linkage mechanism of the art festival.

to promote the win-win goal is necessary to protect the natural environment, make the best use of the situation, and tap potential. In the industrial chain, the life chain will work. Operating institutions, investment funds, and various modes of operation are all part of the industrial chain. The festival's theme is an ecological chain that will grow and connect to other ecological chains.

4.2. The SSFCM-HPR Algorithm Is Used to Analyze the Dataset. The clustering result of SSFCM-HPR algorithm is shown in Figure 4, and the clustering result is very ideal. It can be seen from Figure 4 that when the supervised samples 
contain outliers, SSFCM-HPR algorithm can handle the supervised outliers well so that it can correctly guide the clustering process.

China's rural revitalization is a road of all-round development, and it is an all-round revitalization of industry, ecology, local civilization, and quality of life. Through industrial implantation, ecological environment governance, and promotion, excavation, and activation of local culture, we can retain the local resident population, let urban people return to their hometowns to start businesses, change the rural population structure, reduce population loss, and improve the civilized quality of the whole rural population. Therefore, "people" are particularly important in the whole rural revitalization.

The IRIS dataset is classified by traditional FCM algorithm, FCM algorithm based on feature weighting, and SSFCM-HPR algorithm. The results are shown in Figure 5.

SSFCM-HPR algorithm has the lowest misclassification rate, and with the increase of the number of supervised samples, the recognition rate is significantly improved [23]. When the number of supervised samples is 7, IRIS datasets can be completely separated correctly.

In order to further illustrate the practicability of this algorithm, two face databases, ORL face database and YALE face database, are used for experiments [24]. PCA [14], LDA [15], LPP [16], and MFA [17] algorithms are used for comparison, and the experiments are repeated for 20 times. Figure 6 shows the average recognition rate of various algorithms.

SSFCM-HPR algorithm can better guide the clustering process. Of course, compared with SSFCM-HPR algorithm, FCM_DNE algorithm also has its own characteristics: FCM_DNE algorithm can effectively alleviate the dimension disaster problem in high-dimensional data classification, and at the same time, it maps data to low-dimensional space, which reduces the computational cost of FCM algorithm.

In order to further illustrate the robustness of this algorithm, now, discuss the situation when the label information of the supervision sample is wrong. For the data provided by Kong et al. [18], when labeling the supervision samples, this paper specially gives the supervision samples with wrong classification information. The 2nd, 10th, 41st, and 55th special samples are taken as supervised samples, and two misclassified samples are produced by using Bensaid-Bezdek algorithm. Using SSFCM-HPR algorithm, the result is shown in Figure 7, and there is no misclassification sample. The experiment shows that this algorithm is insensitive to the "typicality" of supervised samples.

Figure 8 shows the membership degree of each sample belonging to the first class when the $\alpha$-value takes some representative values. Obviously, when $\alpha$ is 5 , the ideal classification result is not obtained, and when $\alpha$ is 6 or above, the classification result is ideal.

Although the supervised sample's membership value approaches the labeled membership value as the scale factor is increased, the clustering results of the SSFCM-HPR algorithm are very sensitive to the value of the scale factor, and when the value is small, the role of the supervised sample cannot be displayed, and the clustering results tend to fall into a local optimum, resulting in clustering failure. The

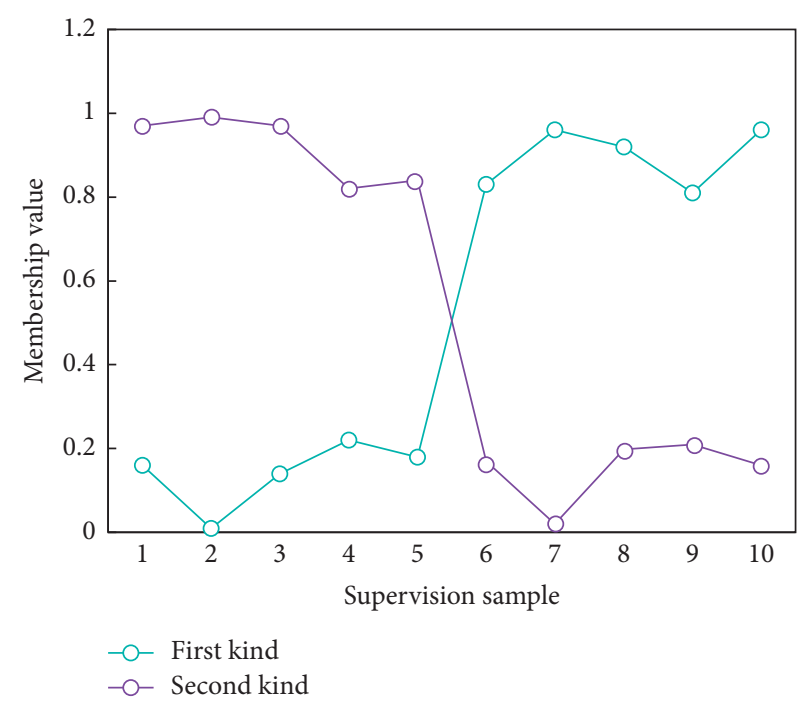

FIGURE 4: Classification results of datasets by SSFCM-HPR algorithm.

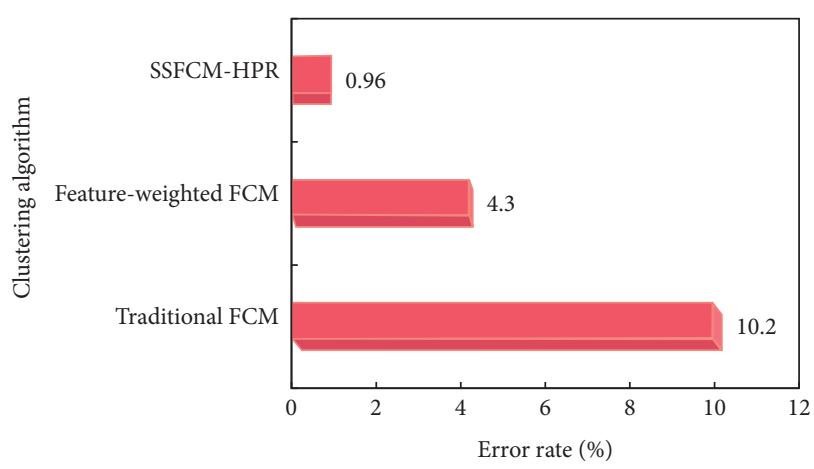

FIGURE 5: Clustering results of different algorithms.

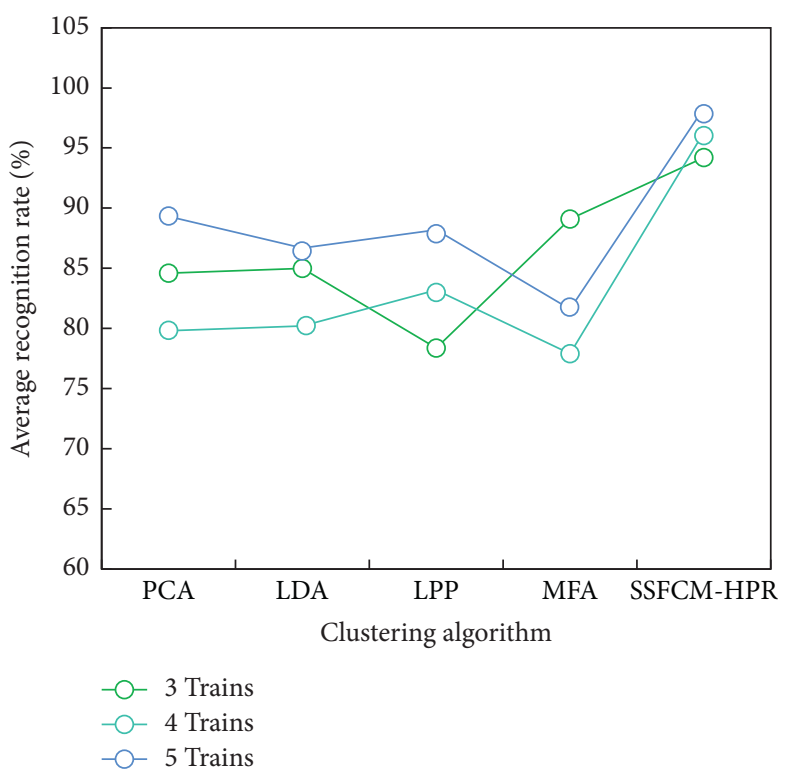

FIgURE 6: Average recognition rate of various algorithms on the ORL database. 


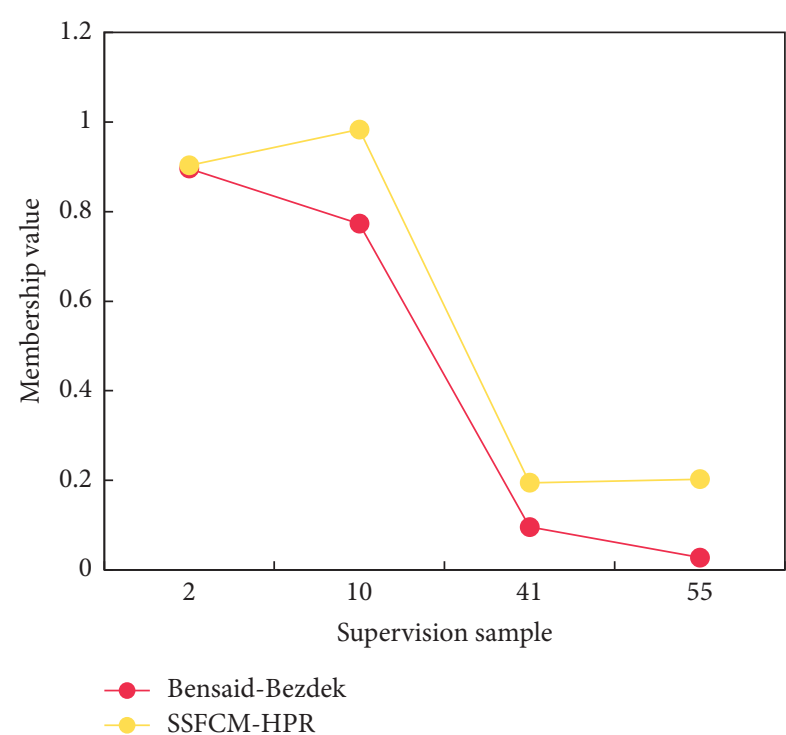

Figure 7: Comparison of weights and membership values of supervised samples by each algorithm.

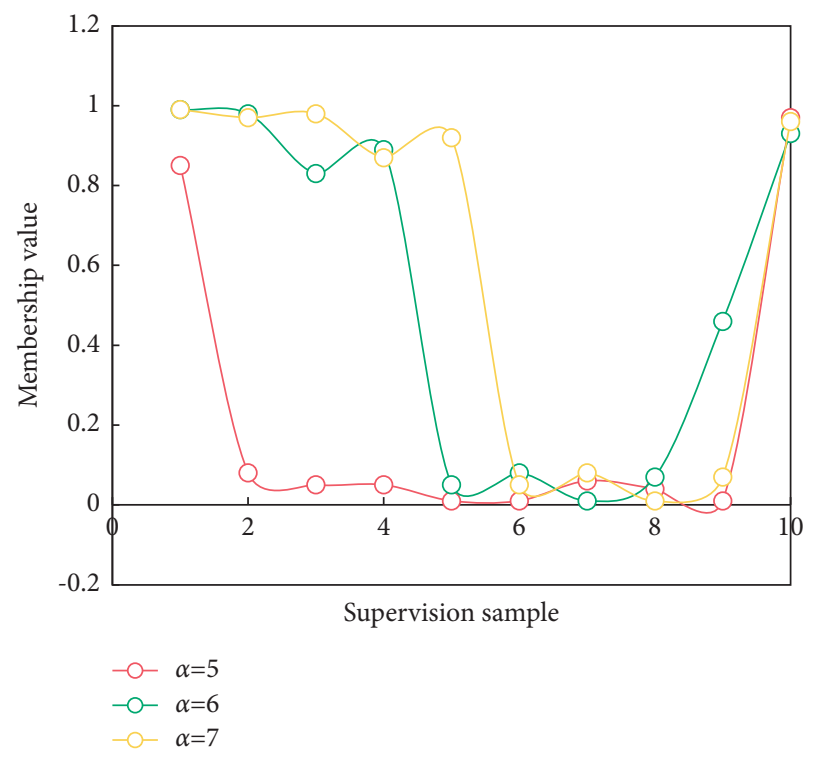

Figure 8: Dividing value of the membership degree of each sample.

weight of unsupervised samples increases as the value increase, weakening the guiding role of supervised samples in the clustering process.

4.3. The Analysis of FCM-UserCF Algorithm. This paper devised a performance test experiment to determine the efficacy of the FCM-UserCF algorithm. The experimental dataset for this study is MovieLens. The data come from a nonprofit research-oriented web recommendation system website. The dataset has two main components: a large number of users and a large number of movies, and the users' movie rating data are sufficient. The experimental dataset includes a training dataset and a test dataset, similar to other types of machine learning datasets. The training set-to-test set

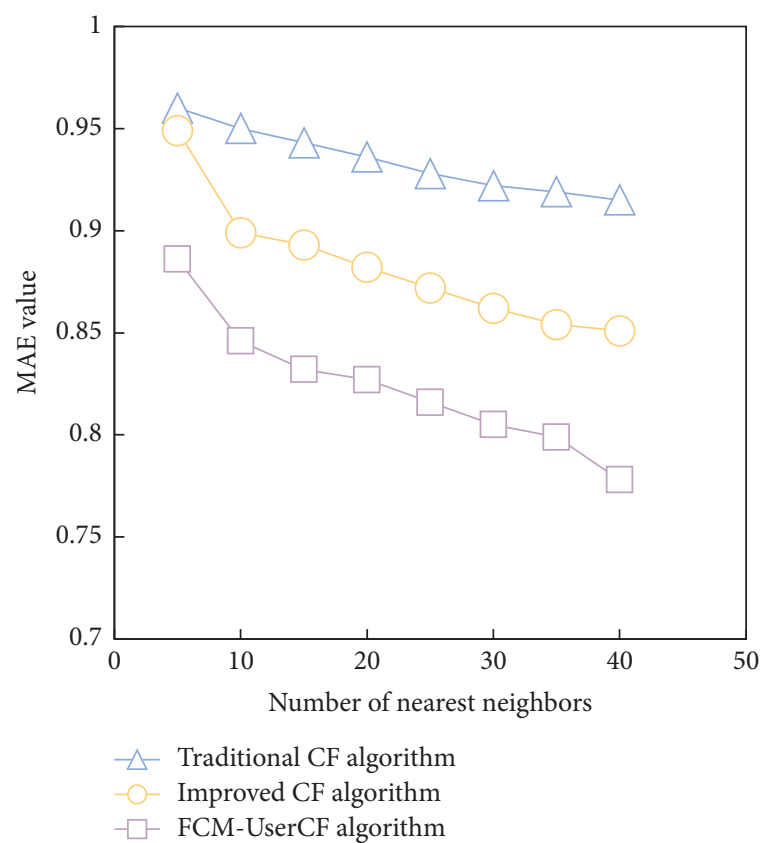

FIGURE 9: Comparison of algorithm effects.

ratio is $8: 2$. The MAE (mean absolute error) experiment is used to assess the algorithm's effectiveness. The MAE value is inversely proportional to the recommendation quality because it is the average of the absolute values of errors between all individual user ratings and real ratings.

Comparing the parallel FCM-UserCF algorithm, the improved CF algorithm proposed in [20], and the traditional $\mathrm{CF}$ algorithm, when calculating the average absolute error value, the number of nearest neighbors takes 5 as the starting value and increases to 30 at intervals of 5 . Figure 9 compares the effects of the algorithms.

In this paper, considering that users with similar backgrounds are more likely to have similar tastes in life, FC is carried out according to user background information, and similarity calculation is carried out among users with similar backgrounds. Moreover, the Slope One algorithm is used to fill the missing data before $\mathrm{CF}$, which is conducive to better selecting the nearest neighbor, thus improving the accuracy of recommendation.

In this paper, experiments are carried out with different scales of data, and the time consumption of parallelizing FCM-UserCF algorithm and serializing FCM-UserCF algorithm under different scales of data is compared. The calculation time of the data scale from 50,000 to 300,000 is shown in Figure 10.

Serialized FCM-UserCF algorithm is more time-consuming than parallelized FCM-UserCF algorithm, which is due to the large memory consumption in FC and similarity matrix calculation, and such intensive calculation is also the shortcoming of the CPU.

In contrast, the parallelized FCM-UserCF algorithm adopts a distributed form when dealing with large amounts of data, and each node calculates at the same time, splitting and merging big data, which also fully embodies the advantages of parallel calculation of master-slave nodes. 


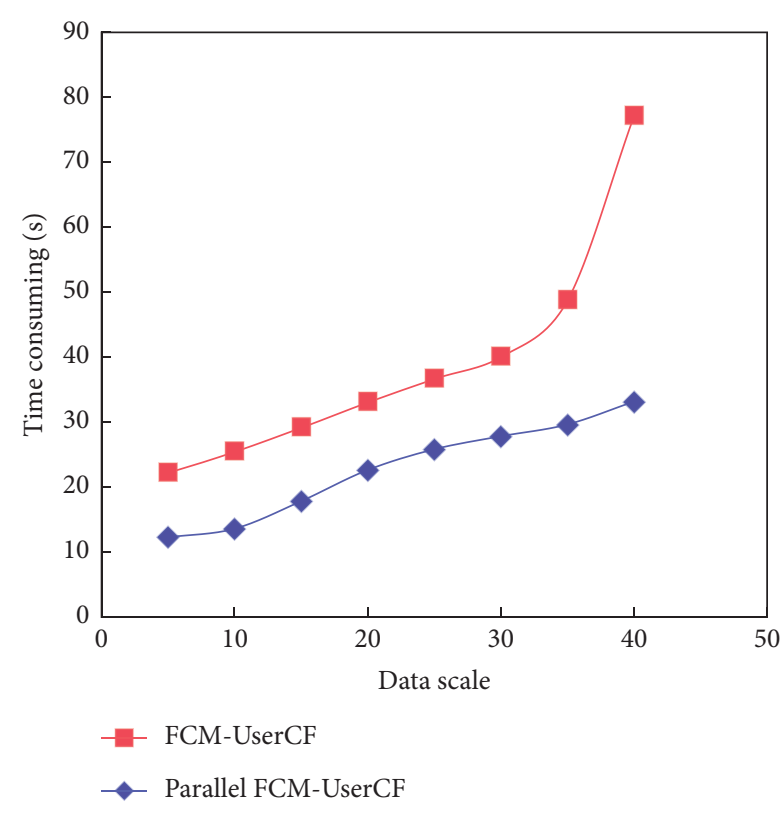

Figure 10: Time consumption comparison.

\section{Conclusion}

The preservation and transmission of concrete material culture, as well as the preservation and transmission of its cultural subject and lifestyle, are all part of the reconstruction of local culture. Art's role in rural revitalization offers distinct benefits in terms of creation design, ingenuity culture, cultural preservation and dissemination, and so on. This paper introduces a novel SSFCM-HPR algorithm. This algorithm not only preserves the fuzziness of supervised sample division but also better reflects the clustering process's guiding role of various supervised samples. It should be treated with FCM-UserCF. The analysis of system requirements, the design of the system architecture and functions, and the design of the database are all completed. The system interface's realization effect is shown, and the function test is completed. The test results demonstrate that the work presented in this paper is practical. Recognize the value and importance of rural culture in the development of urbanization and modernization, and use proper protection and inheritance methods to ensure that rural revitalization continues to progress and live forever.

The implementation mechanism, personnel organization structure, and funding sources required by art intervention in rural planning and design were not discussed in depth in this study. As a result, the study's main findings must be continuously practiced and verified in the future.

\section{Data Availability}

The data used to support the findings of this study are included within the article.

\section{Conflicts of Interest}

The author declares no conflicts of interest.

\section{Acknowledgments}

This study was supported by Guangdong Special Project in Key Areas for Colleges and Universities (Science and Technology Serving Rural Revitalization), Research on the Theory and Practice of Art-Intervention's Enabling Rural Revitalization from Art Colleges (no. 2021ZDX4062).

\section{References}

[1] H. Zou, "On the involvement of wall painting art in rural revitalization and beautiful rural construction," The Artist, vol. 3, no. 3, p. 2, 2021.

[2] L. Li and L. Qi, "The necessity and form of art's intervention in the countryside-—taking Shijiezi Village as an example," Artwork Jian, vol. 10, no. 10Z, p. 2, 2019.

[3] H. Chai and C. Zheng, "Art intervention in the country: talking from the village construction experience of the "Village-making movement"," Think Tank Times, vol. 1, no. 1, p. 2, 2020.

[4] S. Yuan, "The design intervention of farming and folk culture under the background of social transformation and rural revitalization: taking Shanxi Jinnan as an example," Artwork Jian, vol. 12, no. 12, pp. 158-159, 2020.

[5] H. Fan, "Art intervention "lets the country revitalize" art flower road," Journal of the Academy of Management Cadres of the Ministry of Agriculture, vol. 4, no. 4, p. 3, 2020.

[6] R. Xie and S. Pei, "The logical mechanism and realization path of art intervention in the construction of beautiful countryside," Sichuan Drama, vol. 6, no. 6, p. 5, 2020.

[7] Financial Times, "Art and Rural Revitalization," Knowledge Library, vol. 10, no. 10, p. 1, 2018.

[8] W. Zhang and L. Fan, "Research on the significance and methods of art intervention in the country--taking xishu village as an example," Jin Gu Wen Chuang, vol. 13, no. 13, p. 2, 2020.

[9] $\mathrm{S} . \mathrm{Xu}$, "Research on the value of public art intervention in rural construction in my country," China Strategic Emerging Industries, vol. 000, no. 16, pp. 1-3, 2019.

[10] L. Pan, "Development ideas of rural public art," Public Art, no. 6, p. 2, 2019.

[11] F. Zhang, J. Dou, and H. Liang, "Research on public art intervention in rural complex elderly care space," Art and Design: Theory Edition, vol. 8, no. 8, p. 3, 2021.

[12] C. Shang, "Rethinking and design of art township construction intervention in rural industry revitalization," Contemporary Artist, vol. 4, no. 4, p. 6, 2021.

[13] Z. Ying, J. Wang, D. Han, and H. Wu, "Fuzzy-logic based distributed energy-efficient clustering algorithm for wireless sensor networks," Sensors, vol. 17, no. 7, p. 1554, 2017.

[14] S. Ding, M. Du, T. Sun, X. Xu, and Y. Xue, “An entropy-based density peaks clustering algorithm for mixed type data employing fuzzy neighborhood," Knowledge-Based Systems, vol. 133, no. oct.1, pp. 294-313, 2017.

[15] Q. Wang, S. Guo, J. Hu, and Y. Yang, "Spectral partitioning and fuzzy C-means based clustering algorithm for big data wireless sensor networks," EURASIP Journal on Wireless Communications and Networking, vol. 2018, no. 1, p. 54, 2018.

[16] M. E. Aminanto, H. Kim, K.-M. Kim, and K. Kim, "Another fuzzy anomaly detection system based on ant clustering algorithm," IEICE - Transactions on Fundamentals of Electronics, Communications and Computer Sciences, vol. E100.A, no. 1, pp. 176-183, 2017. 
[17] M. G. Karunambigai, M. Akram, S. Sivasankar, and P. Kasilingam, "Clustering algorithm for intuitionistic fuzzy graphs," International Journal of Uncertainty, Fuzziness and Knowledge-Based Systems, vol. 25, no. 3, pp. 367-383, 2017.

[18] X. Kong, Q. Hu, X. Dong, and Y. Zeng, "Load data identification and correction method with improved fuzzy C-means clustering algorithm," Dianli Xitong Zidonghua/Automation of Electric Power Systems, vol. 41, no. 9, pp. 90-95, 2017.

[19] Y. Qu and Y. Wang, "Segmentation of corpus callosum based on tensor fuzzy clustering algorithm," Journal of X-Ray Science and Technology, vol. 29, no. 5, pp. 1-14, 2021.

[20] S. M. Lee, I. Seong, and Y. H. Joo, "Recognition and tracking of moving objects using label-merge method based on fuzzy clustering algorithm," Transactions of the Korean Institute of Electrical Engineers, vol. 67, no. 2, pp. 293-300, 2018.

[21] W. Cai, B. Zhai, Y. Liu, R. Liu, and X. Ning, "Quadratic polynomial guided fuzzy C-means and dual attention mechanism for medical image segmentation," Displays, vol. 70, Article ID 102106, 2021.

[22] Y. Zhang, L. L. Zhai, and M. Shahbaz, "A novel fuzzy clustering approach based on breadth-first search algorithm," Computer Journal, vol. 30, no. 3, pp. 162-175, 2019.

[23] C. Wang, X. Wang, J. Zhang, L. Zhang, X. Bai, and X. Ning, "Uncertainty Estimation for Stereo Matching Based on Evidential Deep Learning," Pattern Recognition, vol. 124, Article ID 108498, 2021.

[24] X. Gu, F. L. Chung, H. Ishibuchi, and S. Wang, "Imbalanced TSK fuzzy classifier by cross-class bayesian fuzzy clustering and imbalance learning," IEEE Transactions on Systems, Man, and Cybernetics: Systems, vol. 47, no. 8, pp. 2005-2020, 2017. 